Z Rheumatol 2021 · 80:756-757

https://doi.org/10.1007/s00393-021-01030-2

Angenommen: 25. Mai 2021

Online publiziert: 25. Juni 2021

(c) Springer Medizin Verlag $\mathrm{GmbH}$, ein Teil von Springer Nature 2021

\section{Redaktion}

C. Fiehn, Baden-Baden

B. Hellmich, Kirchheim-Teck

I. Kötter, Hamburg

K. Krüger, München

\section{Originalpublikation}

Colmegna I et al (2019) Immunogenicity and safety of high-dose versus standard-dose inactivated influenza vaccine in rheumatoid arthritis patients: a randomised, double-blind, active-comparator trial. The Lancet Rheumatology 2(1):E14-E23. https://doi.org/10.1016/ S2665-9913(19)30094-3

Patienten mit rheumatoider Arthritis (RA) tragen ein erhöhtes Risiko für Influenzainfektionen und zeigen, auch wenn sie noch keine immunsuppressive Therapie erhalten, eine gegenüber Gesunden verminderte immunologische Reaktion auf Impfungen [1, 2]. Die Optimierung von Impfantworten bei Patienten mit entzündlich rheumatischen Erkrankungen ist durch die SARSCoV-2-Pandemie von hoher Aktualität. Eine Möglichkeit zur Optimierung der Impfantwort besteht in der Erhöhung der Impfstoffdosis, was im Falle der Influenzaimpfung für über 65-jährige Patienten nachgewiesen wurde [3]. Die Autoren der hier vorgestellten kanadischen Arbeit haben untersucht, ob RA-Patienten von einem höher dosierten Impfstoff gegen Influenza profitieren [4]. Dafür verabreichten sie in einer randomisierten doppelblinden Studie seropositiven RAPatienten den von der Firma Sanofi Pasteur entwickelten trivalenten Impfstoff mit $65 \mu$ g Hämagglutinin (HD-TIV) vs. den quadrivalenten Impfstoff in der Standarddosierung mit $15 \mu \mathrm{g}$ Hämagglutinin (SD-QIV) [3]. Untersucht wurden die

\title{
Michaela Christenn
}

Praxis für Rheumatologie, Schwerpunkt klinische Immunologie, Baden-Baden, Deutschland

\section{Immunogenität und Sicherheit von Hochdosis vs. Standarddosis eines inaktivierten Influenza- impfstoffes bei Patienten mit rheumatoider Arthritis}

Sicherheit sowie die Immunogenität der Hochdosisimpfung im Hämagglutinationshemmtest hinsichtlich der InfluenzaA-Stämme H1N1 und H3N2 sowie des Influenza-B-Stammes Brisbane/60/2008 in 2 Zeiträumen der Jahre 2016 und 2017. Die Autoren verglichen bewusst einen quadrivalenten mit einem trivalenten Impfstoff, um der Population in der SD-Gruppe einen möglichst hohen Impfschutz zu gewährleisten. Ein quadrivalenter Impfstoff in der HD-Dosierung war zum Untersuchungszeitpunkt nicht verfügbar. Dass der quadrivalente $H D$ Impfstoff zusätzlich den Influenza-BStamm B/Phuket/3073/2013 enthielt, wurde als nicht relevant hinsichtlich der Wirksamkeit bezüglich der 3 untersuchten Influenzastämme diskutiert.

Es wurden 279 Patienten hinsichtlich ihres Therapieregimes der letzten 3 Monate in 3 Gruppen unterteilt: Gruppe 1 erhielt csDMARDs als Mono- oder Kombinationstherapie $(n=140)$, Gruppe 2 erhielt TNF- $\alpha$-Inhibitoren oder IL-6-Antagonisten +/- csDMARDs ( $n=48)$, Gruppe 3 erhielt Abatacept, Rituximab oder Tofacitinib +/- csDMARDs $(n=24)$.

Primärer Endpunkt war die Serokonversionsrate an Tag 28 nach Impfung, wobei als Serokonversion ein Anstieg des Hämagglutinationstiters im Mikroneutralisationsassay auf das mindestens 4-Fache des Ausgangstiters definiert war. Als sekundärer Endpunkt galt die Rate an unerwünschten Ereignissen sowie die Seroprotektionsrate als Anteil der
Patienten mit ausreichendem Impftiter von mindestens 1:40 als Surrogatparameter für die Wirksamkeit des jeweiligen Impfstoffes. Die Erkrankungshäufigkeit wurde nicht direkt untersucht. Messzeitpunkte für serologische und klinische Daten waren Tag 0, Tag 28 und Tag 186. Durchschnittlich waren die Patienten der HD-TIV-Gruppe 59,7 Jahre alt (Range 19 bis 87 Jahre), die der SD-QIV-Gruppe 61,9 Jahre alt (Range 24 bis 86 Jahre).

Die Intention-to-treat-Analyse konnte zeigen, dass die Impfung mit HDTIV die Wahrscheinlichkeit einer Serokonversion für alle 3 untersuchten Influenzastämme bis auf den H1N1-Stamm im Jahr 2017 gegenüber der Impfung mit SD-QIV signifikant erhöht (A/H3N2: OR 2,99, $95 \%$-CI 1,46-6,11; A/H1N1: OR 3,21, 1,57-6,56; B/Brisbane: OR 1,95, 1,19-3,22). Die häufigsten unerwünschten Ereignisse waren neu aufgetretene Myalgien, Kopfschmerzen und Müdigkeit, welche jedoch ähnlich häufig über die beiden Dosierungen verteilt waren. Schübe der RA wurden nicht beobachtet. Die verbesserte Immunogenität von HDTIV war zudem über alle Altersgruppen hinweg nachweisbar und nicht nur der älteren Population vorbehalten.

Die Einnahme von Methotrexat (MTX) mit oder ohne Kombination mit b-oder tsDMARD verringerte die Serokonversionsrate nach HD-TIV-Impfung nicht, wenn auch die Aussage durch die geringe Patientenzahl in den verschiedenen Subgruppen ohne MTX limitiert 
war. Auch im Vergleich zwischen Gruppe 1 und 2 fand sich kein signifikanter Unterschied, es konnte für beide Gruppen eine erhöhte Serokonversionsrate nach HD-TIV für alle Stämme außer H1N1 nachgewiesen werden. Eine Aussage für Gruppe 3 war angesichts $\mathrm{zu}$ geringer Fallzahlen nicht möglich.

Die Autoren konnten zeigen, dass der ursprünglich für über 65-Jährige entwickelte Hochdosisimpfstoff im Vergleich zum Standardimpfstoff bei RA-Patienten sowohl über wie auch unter 65 Jahren für alle 3 untersuchten Influenzastämme eine höhere Serokonversions- und Seroprotektionsrate bewirkt, welche auch zum Untersuchungszeitpunkt nach 186 Tagen noch nachweisbar war. Diese Wirkung war unabhängig vom Therapieregime sowohl unter den untersuchten cs- wie auch bDMARDs nachweisbar mit der Limitation, dass eine Aussage für Abatacept, Rituximab und Tofacitinib nicht möglich ist. Eine weitere Limitation ist, dass die Serokonversionsrate natürlich nur ein Surrogatparameter für den Schutz gegen die Erkrankung ist. Das tatsächliche Infektionsrisiko wurde nicht untersucht.

Die Ergebnisse sind konsistent mit vorangehenden Untersuchungen, die einen ähnlichen Effekt für Krebspatienten unter Chemotherapie, Nieren- und Lebertransplantierte und HIV-Patienten zeigen konnten. MTX verringerte den Effekt der HD-TIV gegenüber DMARDnaiven Patienten nicht, ebenso wenig wie b- oder tsDMARDs im Vergleich $\mathrm{zu}$ csDMARD-only-Therapien. Es wird daher postuliert, dass der Benefit der HD-TIV bei RA-Patienten nicht dem Patientenkollektiv einer bestimmten DMARD-Therapie und auch nicht einer bestimmten Altersgruppe vorbehalten bleibt.

Diese deutlichen Effekte zeigen am Beispiel der Influenza, wie bedeutsam eine krankheitsspezifische Anpassung der Impfstrategie für verschiedene chronische Grunderkrankungen sein könnte. Die Forschungslücke ist angesichts verschiedener immunkompromittierender Erkrankungen und Therapieregimes noch groß. Der quadrivalente Hochdosisimpfstoff „Fluzone High-Dose Quadrivalent" ist seit Ende November 2020 für Patienten über 65 Jahre zugelas- sen und bietet damit die Möglichkeit, größere Kohorten an immunkompromittierten Patienten höheren Alters und die Effekte einer Impfdosisanpassung gezielt bezüglich der unterschiedlichen Impfstoffdosen zu vergleichen.

Impfungen gegen SARS-CoV-2 werden im Jahr 2021 entscheidend sein, um die Pandemie zu bekämpfen. Noch ist unbekannt, ob wir bei Patienten mit entzündlich rheumatischen Erkrankungen eine reduzierte Impfantwort befürchten müssen und welche Rolle u. U. die Therapien und das Alter der Patienten dabei spielen könnten. Auch wenn aus der vorliegenden Studie angesichts anderer Wirkstoffmechanismen der zugelassenen SARS-CoV-2-Impfstoffe nicht direkt auf ähnliche Effekte zurückgeschlossen werden kann, erwarten wir mit Spannung die Daten der laufenden Impfstudien diesbezüglich. Die hier vorgestellte Arbeit zeigt exemplarisch, dass es möglich ist, eine Impfung an die immunologischen Bedingungen bei RAPatienten anzupassen.

\section{Korrespondenzadresse}

\section{Dr. med. Michaela Christenn}

Praxis für Rheumatologie, Schwerpunkt klinische Immunologie

Beethovenstr. 2, 76530 Baden-Baden, Deutschland

m.christenn@rheuma-badenbaden.de

Interessenkonflikt. M. Christenn gibt an, dass kein Interessenkonflikt besteht.

\section{Literatur}

1. Chen CM, Chen HJ, Chen WS, Lin CC, Hsu CC, Hsu YH (2018) Clinical effectiveness of influenza vaccination in patients with rheumatoid arthritis. Int J Rheum Dis 21(6):1246-1253. https://doi.org/ 10.1111/1756-185X.13322

2. Wagner N, Assmus F, Arendt G et al (2019) Impfen bei Immundefizienz. Bundesgesundheitsblatt 62:494-515

3. DiazGranados CA, Dunning AJ, Kimmel M, Kirby $D$, Treanor J, Collins A, Pollak R, Christoff J, Earl J, Landolfi V, Martin E, Gurunathan S, Nathan R, Greenberg DP, Tornieporth NG, Decker MD, Talbot HK (2014) Efficacy of high-dose versus standard-dose influenza vaccine in older adults. N Engl J Med 371(7):635-645. https://doi.org/10 1056/NEJMoa1315727

4. Colmegna I et al (2020) Immunogenicity and safety of high-dose vs. standard-dose inactivated influenza vaccine in rheumatoid arthritis patients: a randomized, double-blind, active-comparator trial. Lancet Rheumatol 2:e14-e23
Operationen nach SARSCoV-2-Infektion

Eine neue weltweite Studie des Forschungsnetzwerks COVIDSurg, an dem auch die Universitätsmedizin Halle (Saale) beteiligt ist, zeigt: Operationen sollten nach einer Infektion mit dem Corona-Virus SARS-CoV-2 erst mindestens sieben Wochen nach dem Nachweis stattfinden.

Bereits im Mai 2020 belegten Daten von COVIDSurg, dass die Sterblichkeit von Patientinnen und Patienten bei Operationen nach Coronavirus-Infektionen erhöht ist. In einer aktuellen Veröffentlichung der Fachzeitschrift Anaesthesia nennt das Forschungsnetzwerk nun einen konkreten Zeitraum: Betroffene haben während der ersten sechs Wochen nach einem SARSCoV-2-Nachweis verglichen mit einem später stattfindenden Eingriff ein über zweieinhalbfach erhöhtes Risiko, nach der Operation zu versterben.

Operationen sollten daher entsprechend aufgeschoben werden. Dies gilt auch, wenn nach den sieben Wochen die COVID-19-Symptomatik noch andauert. Bei dringlichen Eingriffen jedoch, wie etwa Tumoroperationen, muss das Risiko eines Fortschreitens der Erkrankung streng gegen das erhöhte Operationsrisiko abgewogen und in bestimmten Fällen auch früher operiert werden.

Das internationale Forschungsnetzwerk COVIDSurg unter der Leitung der Universität Birmingham umfasst über 25.000 Chirurginnen und Chirurgen sowie Wissenschaftlerinnen und Wissenschaftler. Das Projekt ist eine der größten klinischen Studien, die jemals durchgeführt wurden.

Quelle: Universitätsmedizin Halle (Saale), www.medizin.uni-halle.de 